\title{
Differential roles of STAT1 and STAT2 in the sensitivity of JAK2V617F- vs. BCR-ABL- positive cells to interferon alpha
}

\author{
Claudia Schubert ${ }^{1}$, Manuel Allhoff², Stefan Tillmann¹, Tiago Maié2, Ivan G. Costa², Daniel B. Lipka³, \\ Mirle Schemionek ${ }^{1}$, Kristina Feldberg ${ }^{1}$, Julian Baumeister ${ }^{1}$, Tim H. Brümmendorf ${ }^{1}$, Nicolas Chatain ${ }^{1+}$ and \\ Steffen Koschmieder ${ }^{1 * \dagger}$
}

\begin{abstract}
Background: Interferon alpha (IFNa) monotherapy is recommended as the standard therapy in polycythemia vera (PV) but not in chronic myeloid leukemia (CML). Here, we investigated the mechanisms of IFNa efficacy in JAK2V617F- vs. BCR-ABL-positive cells.

Methods: Gene expression microarrays and RT-qPCR of PV vs. CML patient PBMCs and CD34+ cells and of the murine cell line 32D expressing JAK2V617F or BCR-ABL were used to analyze and compare interferon-stimulated gene (ISG) expression. Furthermore, using CRISPR/Cas9n technology, targeted disruption of STAT1 or STAT2, respectively, was performed in 32D-BCR-ABL and 32D-JAK2V617F cells to evaluate the role of these transcription factors for IFNa efficacy. The knockout cell lines were reconstituted with STAT1, STAT2, STAT1Y701F, or STAT2Y689F to analyze the importance of wild-type and phosphomutant STATs for the IFNa response. ChIP-seq and ChIP were performed to correlate histone marks with ISG expression.
\end{abstract}

Results: Microarray analysis and RT-qPCR revealed significant upregulation of ISGs in 32D-JAK2V617F but downregulation in 32D-BCR-ABL cells, and these effects were reversed by tyrosine kinase inhibitor (TKI) treatment. Similar expression patterns were confirmed in human cell lines, primary PV and CML patient PBMCs and CD34+ cells, demonstrating that these effects are operational in patients. IFNa treatment increased Stat1, Stat2, and Irf9 mRNA as well as pY-STAT1 in all cell lines; however, viability was specifically decreased in 32D-JAK2V617F. STAT1 or STAT2 knockout and reconstitution with wild-type or phospho-deficient STAT mutants demonstrated the necessity of STAT2 for IFNa-induced STAT1 phosphorylation in BCR-ABL- but not in JAK2V617F-expressing cells. STAT1 was essential for IFNa activity in both BCR-ABLand JAK2V617F-positive cells. Furthermore, ChIP experiments demonstrate higher repressive and lower active chromatin marks at the promoters of ISGs in BCR-ABL-expressing cells.

Conclusions: JAK2V617F but not BCR-ABL sensitizes MPN cells to interferon, and this effect was dependent on STAT1. Moreover, STAT2 is a survival factor in BCR-ABL- and JAK2V617F-positive cells but an IFNa-sensitizing factor solely in 32DJAK2V617F cells by upregulation of STAT1 expression.

Keywords: IFNa, BCR-ABL, JAK2V617F, MPN, Polycythemia vera, Chronic myeloid leukemia, STAT factors, ISG, CRISPR/Cas9

\footnotetext{
*Correspondence: skoschmieder@ukaachen.de

${ }^{+}$Nicolas Chatain and Steffen Koschmieder contributed equally to this work.

1'Department of Hematology, Oncology, Hemostaseology, and Stem Cell

Transplantation, Faculty of Medicine, RWTH Aachen University, Pauwelsstr 30,

52074 Aachen, Germany

Full list of author information is available at the end of the article
}

(C) The Author(s). 2019 Open Access This article is distributed under the terms of the Creative Commons Attribution 4.0 International License (http://creativecommons.org/licenses/by/4.0/), which permits unrestricted use, distribution, and reproduction in any medium, provided you give appropriate credit to the original author(s) and the source, provide a link to the Creative Commons license, and indicate if changes were made. The Creative Commons Public Domain Dedication waiver (http://creativecommons.org/publicdomain/zero/1.0/) applies to the data made available in this article, unless otherwise stated. 


\section{Background}

Myeloproliferative neoplasms (MPN) are chronic malignancies that are closely associated with specific oncogenes such as BCR-ABL, present in chronic myeloid leukemia (CML) [1], and JAK2V617F, present in polycythemia vera (PV) [2], essential thrombocythemia (ET), and primary myelofibrosis (PMF) [3].

Since 2001, imatinib is the approved standard therapy for treatment of CML that targets differentiated cells $[4,5]$. Imatinib treatment induces a complete cytogenetic response and increases survival and freedom from progression [6]. The JAK1 and JAK2 inhibitor ruxolitinib was found to reduce spleen volume and to improve disease-related symptoms in patients with myelofibrosis $[7,8]$ and hydroxyurea-intolerant or hydroxyurea-resistant PV [9]. However, both imatinib and ruxolitinib do not eradicate CML or MPN stem cells in the majority of cases $[10,11]$. Conversely, both CML and PV stem cells have recently been demonstrated to be targeted by interferon alpha (IFNa) [12-14], but it is not well understood which pathways are responsible for the IFNa response in both diseases.

IFNa induces phosphorylation of signal transducers and activators of transcription 1 (STAT1) and 2 (STAT2) that assemble with interferon-regulatory-factor 9 (IRF9) to form the IFN-stimulated gene factor 3 (ISGF3) complex, which binds to ISRE (IFN-stimulated response element) consensus sequences of their target genes [15]. Although the STAT1-dependent pathway may be the dominant signaling cascade to mediate the antiproliferative effect of IFNa, STAT3 and STAT5 play a role in IFN signaling as well, if only in case of suppressed or abrogated STAT1 signaling [16]. This mechanism may be relevant in CML, since it was shown that cells of IFNa-resistant patients lack STAT1 expression [17] and in PV, when STAT1 function is inhibited by ruxolitinib. Moreover, the JAK/STAT-negative regulator suppressor of cytokine signaling 3 (SOCS3) was upregulated in 50\% of CML patients that did not respond to IFNa [18], and BCR-ABL was shown to suppress the expression of IFNa target genes, thereby modulating the IFNa-mediated response $[19,20]$.

In addition to the JAK/STAT pathway, other signaling pathways are activated by IFNa, such as the p38 mitogen-activated protein kinase (MAPK) pathway [21]. Activation of p38 MAPK has been shown to be responsible for IFNa-induced growth inhibition in IFNa-sensitive CML cells [22] as well as in primary CD34+ cells from PV patients [23]. Furthermore, ULK1 as a trigger of IFNa-induced p38 MAPK signaling in $\mathrm{Ph}^{+}$MPN cells and MEK/ERK-mediated Mnk activation in JAK2V617F-positive cells were described to be required for IFNa-induced growth inhibition [22-25]. Importantly, BCR-ABL expression leads to the degradation of the IFNa receptor (IFNAR) by ubiquitination that could be counteracted by pretreatment of CML cells with imatinib, restoring IFNa sensitivity [26]. But even though IFNAR is downregulated during IFNa therapy [27], this was found to be independent from IFNa responsiveness. Thus, while some IFNa target genes and proteins may be shared between CML and $\mathrm{PV}$, it remains unclear whether the same mechanisms are important for IFNa-induced biologic effects.

In this study, we analyzed the cellular and molecular response of BCR-ABL- and JAK2V617F-expressing cells to IFNa, aiming to clarify the roles of STAT1 and STAT2 in IFNa monotherapy in CML and PV.

\section{Methods \\ Patient samples}

Primary patient samples were obtained from patients of the Department of Hematology, Oncology, Hemostaseology, and Stem Cell Transplantation at RWTH Aachen University after written informed consent, as approved by the local ethics committee (EK 127/12 and EK 206/ 09). The CML patients were newly diagnosed. None of the PV patients had received ruxolitinib or IFNa before sample preparation.

\section{DNA constructs and vectors}

The cDNA of Stat1 was amplified from cDNA of 32D-JAK2V617F, and Stat2 cDNA was ordered from GeneArt gene synthesis service of Thermo Fisher Scientific. Amplification was done with the primers listed in Additional file 1: Table S1. The PCR products were ligated into the pMSCV-IRES-RFP vector digested with the depicted restriction enzymes (NEB) (Additional file 1: Table S1).

Mutagenesis of STAT1 and STAT2 to substitute Y701 (STAT1) or Y689 (STAT2) to phenylalanine (F) was performed with the Quick change II XL site-directed mutagenesis kit (Agilent Technologies) or Q5 mutagenesis kit (NEB) according to the manufacturer's protocol with primers listed in Additional file 1: Table S2. Successful mutagenesis was controlled by Sanger sequencing (Eurofins Genomics S.A., MWG-Biotech, Ebersberg, Germany).

\section{Cell culture}

The production of viral particles and retroviral transduction of $32 \mathrm{D}$ cells was carried out as previously described [28, 29]. The vectors pMY-IRES-GFP-Bcr-Abl, Jak2V617F, or the empty vector (EV) were used for the production of virus. 32D-BCR-ABL or 32D-JAK2V617F cells grow IL-3 independent and can be selected by fluorescence-activated cell sorting (FACS) for GFP expression. After transduction with the pMSCV-IRES-RFP vectors for the overexpression of the STAT proteins, the cells were FACS-sorted for double positivity of GFP and RFP with an Aria cell sorter (BD Bioscience, Heidelberg, Germany). 
The cell lines 32D, K562 (BCR-ABL positive), and HEL (homozygously JAK2V617F positive) were purchased from the German collection of microorganisms and cell cultures (DSMZ, Braunschweig, Germany) and cultured in RPMI-1640 medium with 10\% FCS and 1\% PenStrep. WEHI supernatant (10\%) served as IL-3 source for culturing 32D cells.

\section{Real-time quantitative reverse transcriptase-PCR (RT- qPCR)}

RNA isolation, cDNA transcription, and procedure of RT-qPCR were performed as previously described [28]. In general, 32D cells were grown in WEHI-supplemented (IL-3-source) RPMI medium. Sixteen hours before RNA isolation, 32D cells were washed twice and resuspended in IL-3-free medium. Expression was calculated as a percentage of Gapdh (Glycerinaldhyd-3-phosphate-dehydrogenase). The primers for the analysis of target genes are listed in Additional file 1: Table S3.

For microarray analysis, RNA from 32D-EV, 32D-BCR$\mathrm{ABL}$, or 32D-JAK2V617F cells was analyzed, using an Affymetrix mouse genome 4302.0 array (Fisher Scientific, Life Technologies, Carlsbad, CA, USA).

\section{SDS-Page and immunoblotting}

Western blot analysis was performed as previously described [28], and proteins were detected via chemiluminescence (Fusion SL, PeqLab, Erlangen, Germany). The used antibodies are listed in Additional file 1: Table S4.

\section{MTT assay}

Cells were plated in triplicate $\left(5 \times 10^{4}\right.$ cells/well $)$ in WEHI-free medium. IFNa (Miltenyi Biotech; mouse IFNa, source: HEK293 cells; batches changed during the study) was diluted in cell culture medium $\left(0-10^{4} \mathrm{U} / \mathrm{ml}\right)$. In case of combination treatment with TKI, the cells were treated with 0.1 to $1 \mu \mathrm{M} \mathrm{TKI}$, as indicated, in addition to the different IFN concentrations in comparison to a DMSO control. The measurement of cell viability was performed $72 \mathrm{~h}$ later using MTT reagent (Sigma-Aldrich), and measurement of absorption was conducted at a wavelength of 550 $\mathrm{nm}$. The metabolic process of tetrazolium reduction to formazan in our assay reflects the amount of viable cells, and this is therefore stated accordingly. The viability was calculated in reference to the control cells.

\section{Apoptosis staining}

Apoptosis staining of cells after TKI $(0.5 \mu \mathrm{M}$ imatinib and $0.1 \mu \mathrm{M}$ ruxolitinib) and/or IFNa treatment $\left(10^{2} \mathrm{U} /\right.$ $\mathrm{ml}$ ) was done with propidium iodide (Sigma-Aldrich). $1 \times 10^{6}$ cells were treated for $72 \mathrm{~h}$ or $48 \mathrm{~h}$ and stained with Zombie-Aqua or PI, respectively, in a 1:1000 dilution. The samples were analyzed with a FACS Gallios (Beckmann Coulter, Krefeld, Germany).

\section{CRISPR/Cas9n triggered STAT1 or STAT2 knockout}

32D-BCR-ABL-S1ko or S2ko and 32D-JAK2V617F-S1ko or S2ko cells were generated with CRISPR/Cas9n double nicking approach as described by Ran et al. [30]. In brief, two pairs of guide RNA (gRNA) were designed for excision of exon 5-7 in the Stat1 and exon 9-20 in the Stat2 gene (Additional file 1: Table S5; Additional file 2: Figure S1). gRNA oligonucleotides were cloned into a variant of the pX462 vector (Addgene plasmid \# 62987), which was a gift from Feng Zhang (Addgene plasmid \# 62987) [30]. Five micrograms of each gRNA plasmid, targeting STAT1 or STAT2, was electroporated into 32D-BCR-ABL or 32D-JAK2V617F cells using the NEON transfection system $(1.350 \mathrm{~V}, 20 \mathrm{~ms}, 2$ pulse, Thermo Fisher Scientific). Electroporated cells were selected by puromycin treatment $(3 \mu \mathrm{g} / \mathrm{ml})$ for $24 \mathrm{~h}$. Again $24 \mathrm{~h}$ later, single cell dilutions were performed to obtain single clones.

\section{Chromatin immunoprecipitation}

Chromatin immunoprecipitation (ChIP) experiments were conducted as previously described [31]. Antibodies and primers are listed in Additional file 1: Tables S6 and S7, respectively. Results were generated in at least two independent experiments.

Two different sets of H3K9ac ChIP-seq data were analyzed. One was published before [32] and included 32D-EV, 32D-BCR-ABL, and 32D-JAK2V617F cells. The other one was performed by our group in collaboration with the German Cancer Research Center (DKFZ) in Heidelberg in the Division of Epigenomics and Cancer Risk Factors and additionally included the corresponding TKI treatment. The detailed procedure is described in the supplementary information.

\section{Bioinformatic analyses}

Analysis of expression arrays was based on Bioconductor, and analysis of ChIP-seq data was based on differential peak caller THOR [33] to detect peaks gained/lost between previously described pairs of conditions. Gene expression data from patients with BCR-ABL mutations and respective controls were obtained from Gene Expression Omnibus (GSE5550) [34]. The detailed analysis procedure is given in supplementary information. Gene set enrichment analysis (GSEA) was performed with the bionconductor package piano [35]. All gene sets from the Molecular Signatures Database (https://software. broadinstitute.org/gsea/msigdb/) and a gene set with interferon-stimulated genes (ISG) obtained from Wright et al. [36] (see Additional file 1: Table S8) were used. The GSEA test indicates the enrichment of gene sets in either upregulated or downregulated genes based on the fold changes contrasting 32D cells with BCR-ABL vs JAK2V617F, JAK2V617F vs EV, and BCR-ABL vs EV. 


\section{Statistical analysis}

Statistical analysis was performed with GraphPad Prism software 5.0 (GraphPad Software, La Jolla, CA, USA) and the two-tailed Student's $t$ test and one-way ANOVA (Dunnett's test). Significant differences were defined as ${ }^{*} p<0.05$, ${ }^{* * *} p<0.01$, and ${ }^{* * * *} p<0.001$. Mean and standard deviation (SD) are indicated. All experiments have been performed at least three times, if not indicated differently.

\section{Results}

JAK2V617F- and BCR-ABL-positive cells are differentially targeted by IFNa and show opposite effects on interferon-stimulated gene (ISG) expression

The effect of in vitro treatment with IFNa $\left(0-10^{4} \mathrm{U} / \mathrm{ml}\right)$ on cell viability was analyzed in murine $32 \mathrm{D}$ cells, stably expressing BCR-ABL or JAK2V617F. 32D-EV (empty vector) cells served as maternal non-oncogene-expressing control, and cell viability was not altered by increasing IFNa concentrations (Fig. 1a). Viability of 32D-JAK2V617F cells was significantly reduced, while it remained essentially unchanged in 32D-BCR-ABL cells. Combination of IFNa with low doses of imatinib (IM $0.1 \mu \mathrm{M}$ ) sensitized 32D-BCR-ABL cells to IFNa and viability decreased in a concentration-dependent manner (Additional file 3: Figure S2). The viability of 32D-JAK2V617F cells was decreased to approximately 75\% of control with $0.1 \mu \mathrm{M}$ ruxolitinib alone (Rux), but there was no additive effect in combination with IFNa.

Gene expression profiling (GEP) of 32D-EV 32DBCR-ABL, and 32D-JAK2V617F cells demonstrated that ISGs were differentially regulated by the two oncogenes (Additional file 4: Figure S3). This was not an unspecific effect, as illustrated by gene set enrichment analysis (GSEA), showing that IL2/ STAT5 pathway-associated genes were similarly regulated by both oncogenes (Fig. 1b; Additional file 1: Table S8). Validation of Stat1, Stat2, Irf1, Irf7, and Irf9 mRNA expression by RT-qPCR showed a significant downregulation of Stat1, Stat2, and Irf9 in 32D-BCR-ABL cells in comparison to EV cells, while all five analyzed genes were significantly upregulated in 32D-JAK2V617F cells (Fig. 1c, upper panel). Imatinib and ruxolitinib reverted $\mathrm{BCR}-\mathrm{ABL}-$ and JAK2V617F-induced changes, respectively (Fig. 1c, upper panel). These data were confirmed in human cell lines K562 (BCR-ABL-positive) and HEL (JAK2V617F-positive) (Fig. 1c, lower panel). Stat2 expression was high in 32D-BCR-ABL cells and did not change upon imatinib treatment. Using 32D cell supernatants, we were able to exclude that the observed effects on ISG expression were due to soluble factors produced by JAK2V617F-positive cells, as collected supernatants were not capable to induce mRNA expression of the ISGF3 components (Additional file 5: Figure S4).
In order to analyze the impact of BCR-ABL and JAK2V617F on ISG mRNA expression in primary cells, peripheral blood samples from healthy donors, BCR-ABL-positive CML patients, and JAK2V617F-positive PV patients were tested. The expression of all analyzed genes was significantly reduced in the CML samples, while no significant changes were observed in the PV samples (Fig. 1d) except for STAT1 expression, which was significantly reduced in PV samples but not to the same extent as in the CML samples. Importantly, IRF1 and IRF9 expression was significantly higher in JAK2V617F- vs. BCR-ABL-positive PBMNCs (Fig. 1d). IFNa responsiveness did not correlate with the ratio of bcr-abl/abl transcripts of the patients (data not shown). However, a significant correlation of JAK2V617F allele burden and ISG induction was observed for IRF9 ( $p=$ 0.0492) (Additional file 6: Figure S5A), suggesting that IRF9 expression reflects the malignant tumor burden in $\mathrm{PV}$. The presence of additional mutations such as TET2 or ASXL1 mutations did not correlate with low expression of ISG genes (Additional file 6: Figure S5A). Importantly, GEP of CD34-positive cells of BCR-ABL-positive [34] and JAK2V617F-positive [37] MPN patients confirmed differential ISG expression patterns (Fig. 1e), observed in our 32D cell model system and the PBMCs, demonstrating that these changes were not simply due to different cell subpopulations or aberrant murine cell physiology.

\section{Combination treatment of IFNa and imatinib increases the IFNa response in BCR-ABL-positive cells, while ruxolitinib blocks IFNa-triggered signaling}

Next, we analyzed the effect of IFNa alone or in combination with either imatinib or ruxolitinib on mRNA expression of ISGs. Both 32D-BCR-ABL and 32D-JAK2V617F cells showed increased expression of Stat1, Stat2, Irf7, and Irf9 after IFNa treatment (Fig. 2a). Moreover, IFNa-induced mRNA expression was further increased by the addition of imatinib in 32D-BCR-ABL cells, while the addition of ruxolitinib reduced IFNa-induced gene expression in 32D-JAK2V617F cells (Fig. 2a). Similar IFNa-induced effects were seen in the human cell lines K562 and HEL, but no additive effect of imatinib in K562 cells was observed (Fig. 2b).

When protein expression and tyrosine phosphorylation of major signaling molecules were analyzed in 32DBCR-ABL and 32D-JAK2V617F cells after IFNa and/or TKI treatment, 32D-JAK2V617F cells showed higher STAT1 protein expression and phosphorylation compared to BCR-ABL-positive cells, and this phosphorylation was inhibited by ruxolitinib (Fig. 2c), suggesting that this was a JAK2V617F-mediated effect. In addition, 32D-EV cells showed STAT1 phosphorylation only after IFNa stimulation, comparable to BCR-ABL-positive cells (Additional file 6: Figure S5B). IFNa treatment increased total STAT1 protein expression and tyrosine phosphorylation 


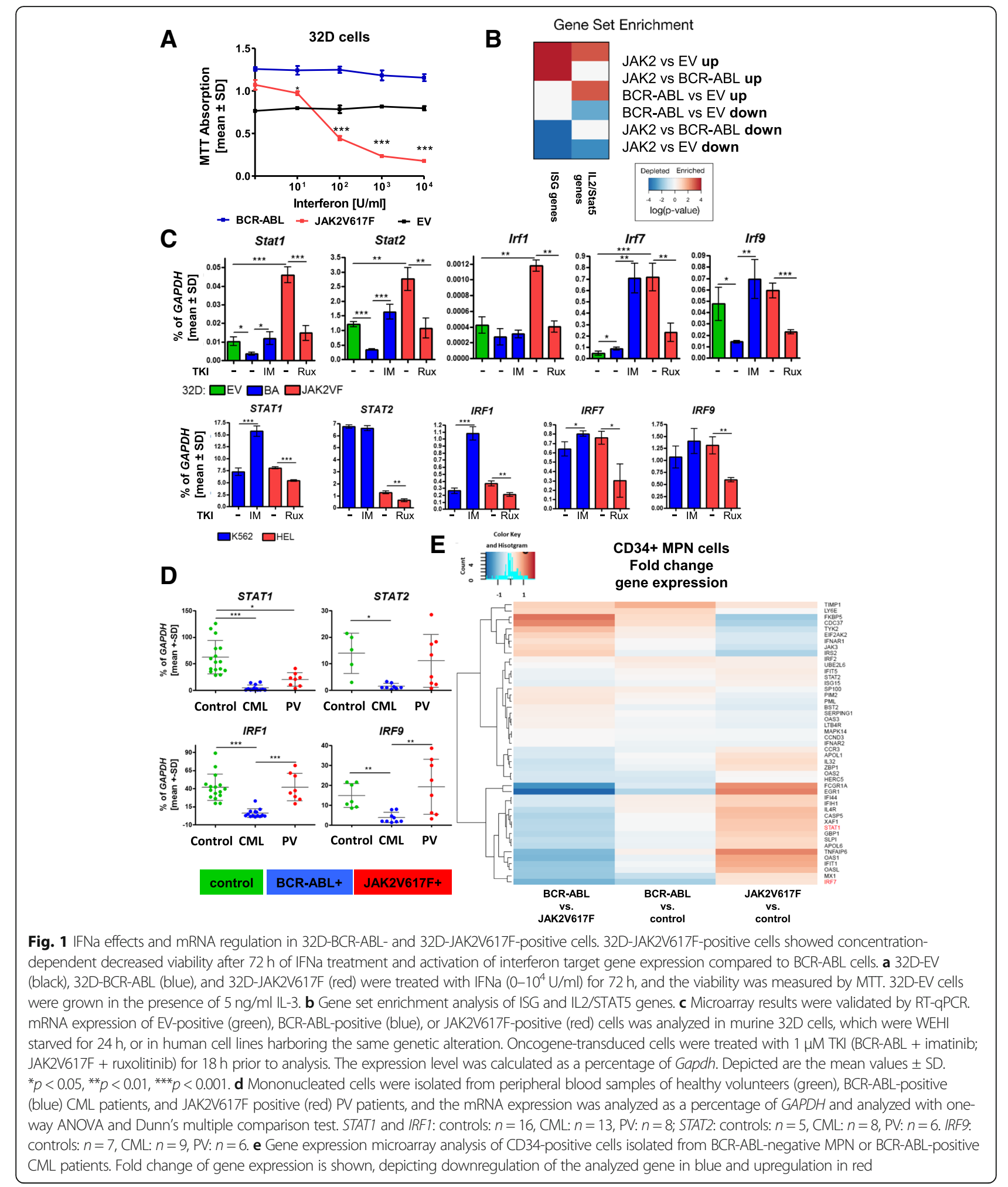

in both BCR-ABL- and JAK2V617F-positive 32D cells and also in K562 and HEL cells (Fig. 2c, d). Interestingly, the combination of imatinib and IFNa further increased the phosphorylation of STAT1 in BCR-ABL-positive cells, while STAT1 phosphorylation was completely blocked in JAK2V617F-positive cells treated with ruxolitinib and IFNa (Fig. 2c, d). We observed differential constitutive activity of STAT2, STAT3, and ERK1/2 phosphorylation 

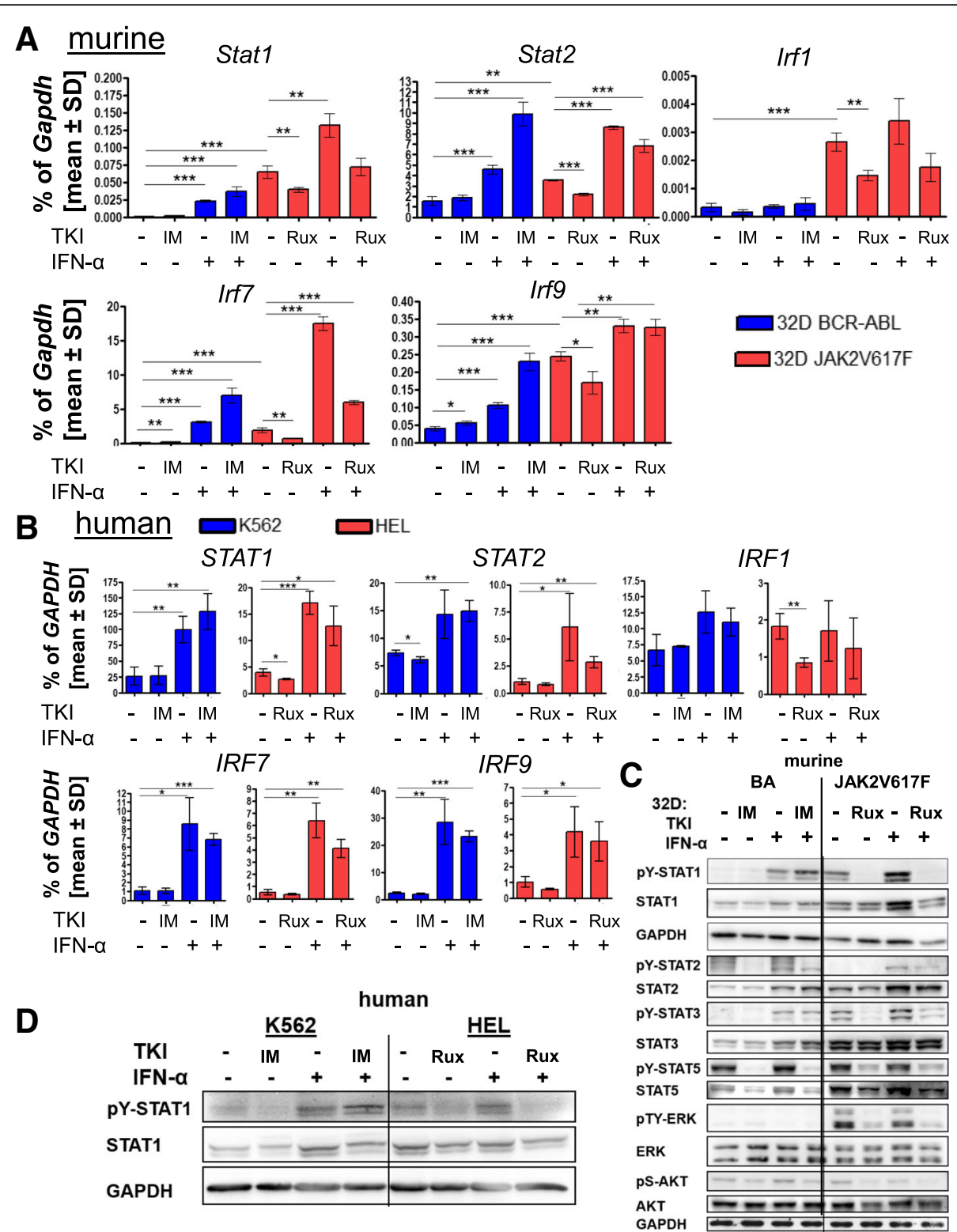

Fig. 2 IFNa and TKl effects on mRNA and protein expression in cell lines and patient PBMCs. BCR-ABL-positive (blue) or JAK2V617F-positive (red) cells were treated with $1 \mu \mathrm{M}$ imatinib (BCR-ABL) or ruxolitinib (JAK2V617F) or $100 \mathrm{U} / \mathrm{ml}$ IFNa or a combination of TKI and IFNa for $4 \mathrm{~h}$. $\mathbf{a}$, b mRNA expression of interferon target genes in transduced murine 32D cells or human K562 (BCR-ABL) or HEL (JAK2V617F) cells, shown as mean values \pm SD as a percentage of GAPDH expression. ${ }^{*} p<0.05,{ }^{* *} p<0.01,{ }^{* * *} p<0.001$. 32D cells were WEHl starved for $24 \mathrm{~h}$ before starting the experiment. $\mathrm{c}$ Western blot analysis of the canonical IFNa and oncogene-triggered signaling after TKI and/or IFNa treatment ( $4 \mathrm{~h}$ ). The indicated antibodies were used, and GAPDH served as the loading control. d STAT1 expression and tyrosine phosphorylation (pY-STAT1) after $4 \mathrm{~h}$ of TKI and/or IFNa treatment in K562 and HEL cells

in 32D-BCR-ABL vs. 32D-JAK2V617F cells (Fig. 2c), with higher pY-STAT2 but lower pY-STAT3, and pTY-ERK1/2 levels in BCR-ABL- than JAK2V617Fpositive cells. All of these were downregulated by the respective TKI, while IFNa-induced pY-STAT1 and PY-STAT3 were downregulated by ruxolitinib but not imatinib.

Thus, both the murine and human cell line results and the data gathered from the primary patient
(PBMC and CD34+) cells demonstrated opposite effects of IFNa +/- TKI in JAK2V617F- vs. BCR-ABLpositive cells.

\section{CRISPR/Cas9-triggered knockout of STAT1 or STAT2 alters} IFNa sensitivity of 32D-JAK2V617F cells

We generated Stat1 and Stat2 knockout 32D-BCR-ABL or 32D-JAK2V617F cells to better define the role of these two STAT proteins in the differential sensitivity to 
IFNa. Several knockout clones were generated with CRISPR/Cas9n technology, verified by Western blotting (Additional file 7: Figure S6), and one clone of each cell line was selected and treated with TKI, IFNa, or the combination (Fig. 3a, b). The results demonstrated that, in BCR-ABL-positive cells, genetic disruption of Stat1 decreased Stat2 protein expression while preserving IFNa-induced STAT2 phosphorylation (Fig. 3a), did not alter viability of untreated cells (Fig. 3c), but abolished the already low IFNa response, with IFNa alone failing to induce apoptosis (Fig. 3c, e). There was no change in ISG expression in the absence of STAT1, suggesting that downregulation of these genes by BCR-ABL, as shown in Fig. 1, was STAT1-independent (Fig. 3f).

In JAK2V617F-positive cells, the absence of STAT1 led to a decrease of STAT2 protein while preserving IFNa-induced STAT2 phosphorylation and to an abrogation of most IFNa-induced effects (ISG expression, reduced cell viability, and with a tendency for induction of apoptosis), suggesting that both the JAK2V617F-induced sensitization to IFNa as well as IFNa-induced effects are STAT1-mediated (Fig. 3b, d-f, and Additional file 8: Figure S9a, b).

The absence of STAT2, in BCR-ABL-positive cells, led to a strong downregulation of STAT1 protein, including induction of pY-STAT1 by IFNa. Irf9 gene expression was significantly downregulated in the absence of STAT2, and there was a trend towards decreased expression of other ISGs, but there was no effect on cell viability or apoptosis, regardless of the presence or absence of IFNa (Fig. 3a, c, e, f).

Conversely, upon STAT2 disruption, IFNa-induced STAT1 phosphorylation was preserved in JAK2V617F cells, with the latter showing nearly complete loss of STAT1 protein (Fig. 3a, b). Also, STAT2 knockout led to a significant reduction of all ISGs in JAK2V617F-positive cells, and these cells showed lower viability and a higher rate of apoptosis (Fig. 3d, e) than their STAT2 wt counterparts. However, IFNa increased viability and decreased, although not significantly, apoptosis in these cells (Fig. 3d, e), suggesting a role of STAT2 in promoting IFNa-induced apoptosis in JAK2V617F cells.

\section{Differential capacities of STAT2 in regulating STAT1 expression depending on the oncogenic background} For a better understanding of the role of unphosphorylated (referring to the regulatory tyrosines Y701 in STAT1 and Y689 in STAT2) STAT1/2 in a BCR-ABL or JAK2V617F background, 32D-BCR-ABLand 32D-JAK2V617F-S1ko or 32D-JAK2V617F-S2ko cells were reconstituted with wt-STAT1, wt-STAT2, the dominant negative form STAT1Y701F (STAT1Y/ F), or the phospho-deficient STAT2Y689F (STAT2Y/F) mutant, respectively.
We observed that STAT2 and STAT2Y/F expression in 32D-BCR-ABL- and 32D-JAK2V617F-S2ko cells led to increased viability in the absence of IFNa treatment (Fig. 4a, b). As expected, STAT1 reconstitution in BCR-ABL- and JAK2V617F-positive STAT1ko cells increased IFNa responsiveness (Fig. 4a, b and Additional file 9: Figure S7A, B). The IFNa response was only slightly reduced in 32D-JAK2V617F-S1ko STAT1Y/ F-expressing cells, also shown in a titration of lower IFNa dosages (Fig. 4a, b and Additional file 9: Figure S7). Interestingly, 32D-JAK2V617F-S2ko-STAT2(Y/F) cells were highly responsive to IFNa treatment (Fig. 4b and Additional file 9: Figure S7), demonstrating that Y689 phosphorylation of STAT2 is not a prerequisite for IFNa efficacy.

In the following, the phosphorylation pattern of STAT1, STAT2, and STAT3 were analyzed to potentially explain the striking differences of STAT2(Y/F) reconstitution in BCR-ABL- and JAK2V617F-positive-S2ko cells. In 32D-BCR-ABL-S1ko cells stimulated with IFNa, STAT1 but also STAT1Y/F expression increased pY-STAT2 as well as the overall STAT2 protein level (Fig. 4c). Therefore, STAT1 but not its phosphorylation at Y701 is necessary for STAT2 expression in BCR-ABLpositive 32D cells. Meanwhile, STAT1Y/F expression in IFNa-stimulated 32D-JAK2V617F-S1ko cells failed to upregulate STAT2 to the same extent (Fig. 4c).

Re-expression of STAT2 as well as STAT2Y/F normalized or even increased endogenous STAT1 expression and phosphorylation in JAK2V617F-positive STAT2ko cells but not in BCR-ABL-positive cells (Fig. 4c, red rectangle). This upregulation in 32D-JAK2V617F-S2ko-STAT2(Y/F) cells could explain the enhanced IFNa sensitivity found in all of our assays (Figs. 1, 2, and 4b). IFNa-induced pY-STAT3 was enhanced by STAT1 disruption and decreased by reconstitution with STAT1 or STAT1Y/F in both cell lines, demonstrating that STAT1 and STAT3 are competing for the same phospho-binding sites at the IFN receptors (Fig. 4c). However, there was a marked difference of IFNa-induced STAT3 phosphorylation in BCR-ABL- and JAK2V617F-positive cells in the absence of STAT2: strong IFNa-induced pY-STAT3 persisted only in JAK2V617Fbut not BCR-ABL-positive cells despite re-expression of STAT2 or the STAT2Y/F mutant (Fig. 4c). In general, STAT3 was only weakly phosphorylated in BCR-ABL vs. JAK2V617F expressing cells (Fig. 2c). Additional file 10: Figure S8 illustrates the differences in PY-STAT1 signaling in BCR-ABL- vs. JAK2V617F-positive cells, shown in Fig. 4c, at higher contrast. The Y/F mutants showed residual pY signals, most likely due to unspecific antibody binding, as the correct sequence of all mutants was confirmed by Sanger sequencing (data not shown).

In the following, ISG expression was further analyzed in reconstituted 32D-STATko cells (Fig. 4d). In 
A

32D-BCR-ABL

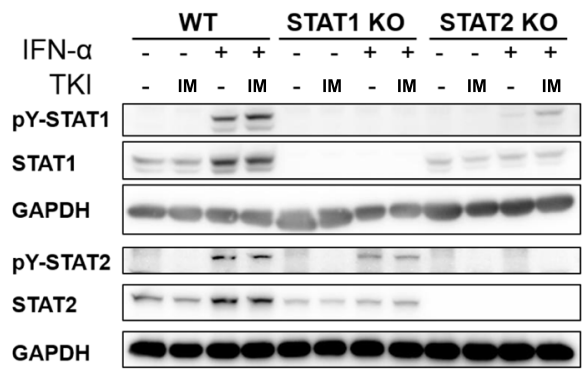

C

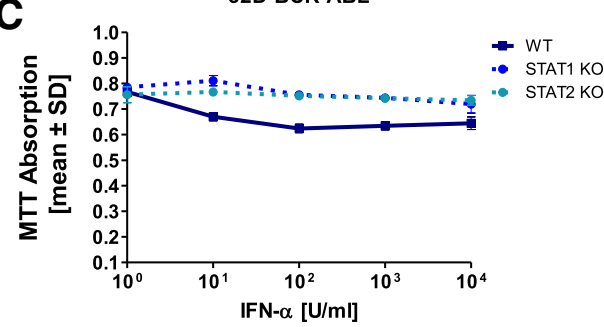

D

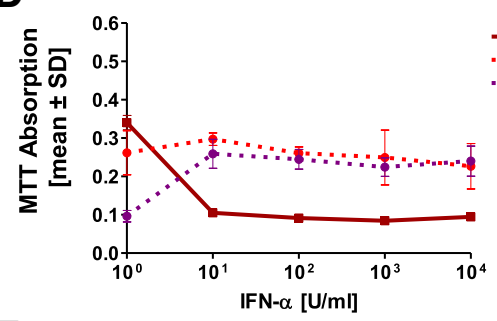

B

\section{D-JAK2V617F}

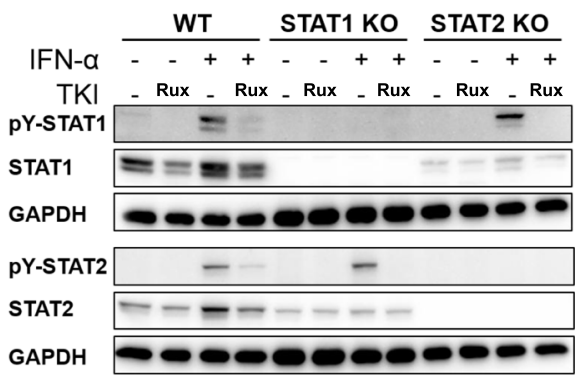

E

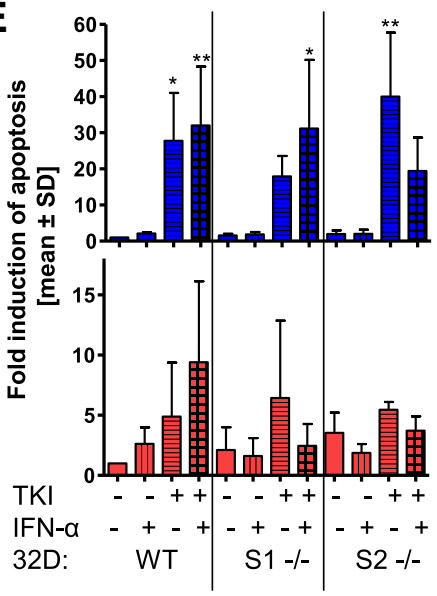

$\square$ 32D BCR-ABL $\square 32 \mathrm{D}$ JAK2V617F

Inthrf7

Ind If 19
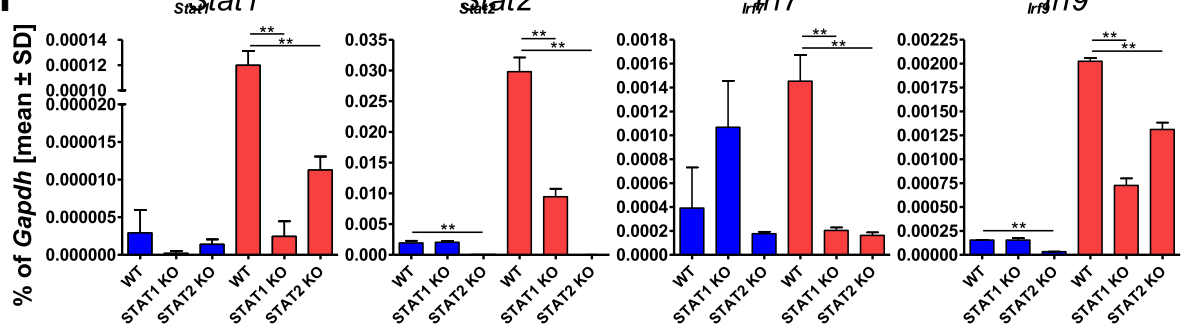

Fig. 3 STAT1 or STAT2 knockout alters IFNa responsiveness only in JAK2V617F-positive cells. a 32D-BCR-ABL-WT, 32D-BCR-ABL-STAT1 ko, and 32D-BCR-ABL-STAT2ko or b 32D-JAK2V617F-WT, 32D-JAK2V617F-STAT1ko, and 32D-JAK2V617F-STAT2ko cells were treated for $4 \mathrm{~h}$ with TKI $(1 \mu \mathrm{M}$ imatinib and ruxolitinib, respectively) or IFNa $(100 \mathrm{U} / \mathrm{ml})$ or a combination of both. SDS-Page and Western blotting were performed, and the indicated immunostainings were carried out. GAPDH served as the loading control. c 32D-BCR-ABL-WT, 32D-BCR-ABLSTAT1 ko, and 32D-BCR-ABL-STAT2ko or d 32D-JAK2V617F-WT, 32D-JAK2V617F-STAT1ko, and 32D-JAK2V617F-STAT2ko cells were treated with increasing concentrations of IFNa, and cell viability was measured by MTT assay. e Indicated 32D cells were treated with TKI $(0.5 \mu \mathrm{M}$ imatinib and $0.1 \mu \mathrm{M}$ ruxolitinib, respectively) or IFNa $(100 \mathrm{U} / \mathrm{ml})$ or a combination of both for $48 \mathrm{~h}$, due to the rapid growth of untreated BCR-ABL-positive cells. PI staining was performed to discriminate between living and dead cells. Mean values \pm SD are depicted. $\mathbf{f}$ Measurement of Stat1, Stat2, Irf7, and Irf9 mRNA expression in the indicated cell lines. Expression was calculated as a percentage of Gapdh, and the mean values \pm SD are depicted. ${ }^{*} p<0.05,{ }^{* *} p<0.01,{ }^{* *} p<0.001$. For all experiments, the respective $32 \mathrm{D}$ cells were WEHI starved for $24 \mathrm{~h}$ before starting the experiments

JAK2V617F-positive cells, expression of all ISGs was fully rescued by STAT1 reconstitution and to a lesser degree by the STAT1Y/F mutant (Fig. 4d), demonstrating STAT1 as one of the key downstream effectors of JAK2V617F in stimulating the IFNa pathway. In BCR-ABL-positive cells, STAT1 reconstitution led to increased Stat1 and Irf9 expression, overriding BCR-ABL-mediated suppression of these genes (Fig. 4d). In the presence of IFNa, ISG expression in STAT1 or STAT2 ko cells was abrogated but strongly induced in STAT1- (and to a lesser extent, STAT1Y/F)-reconstituted 
A

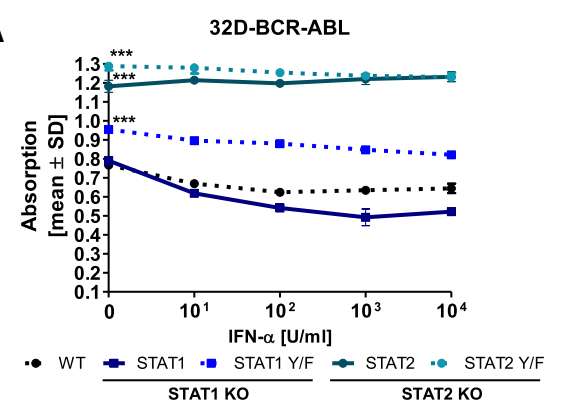

B

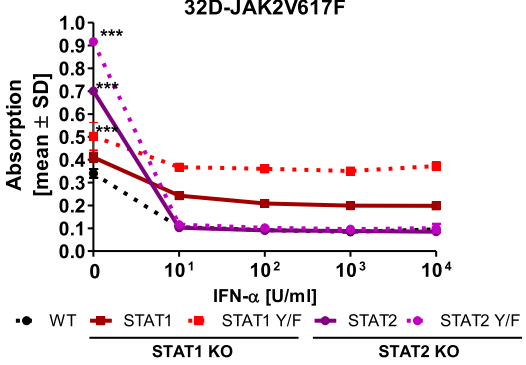

C

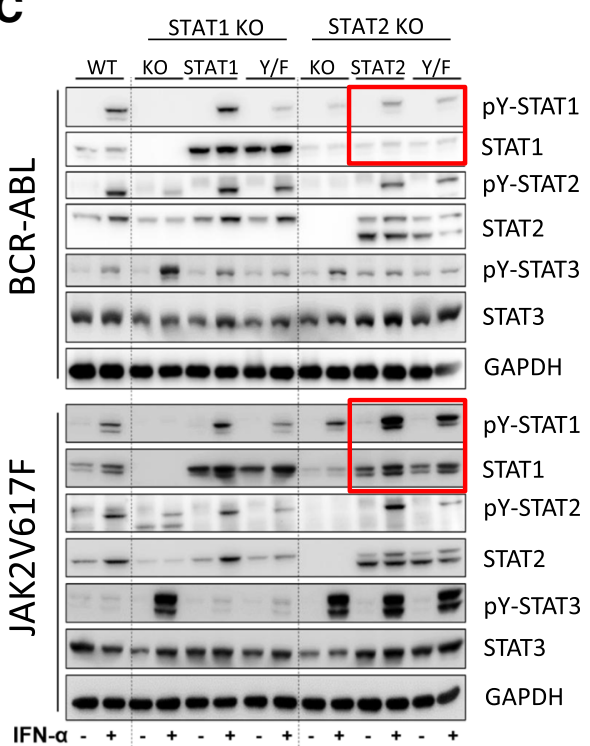

D

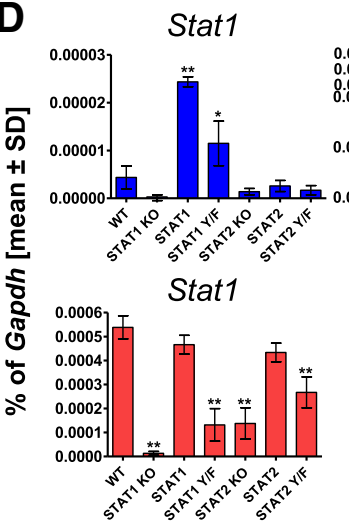

Stat2

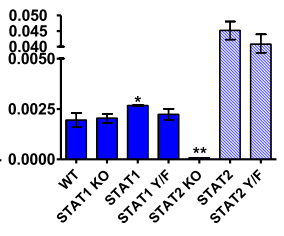

Stat2

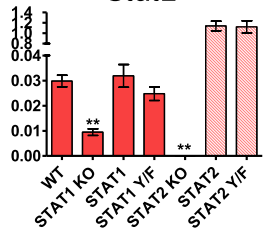

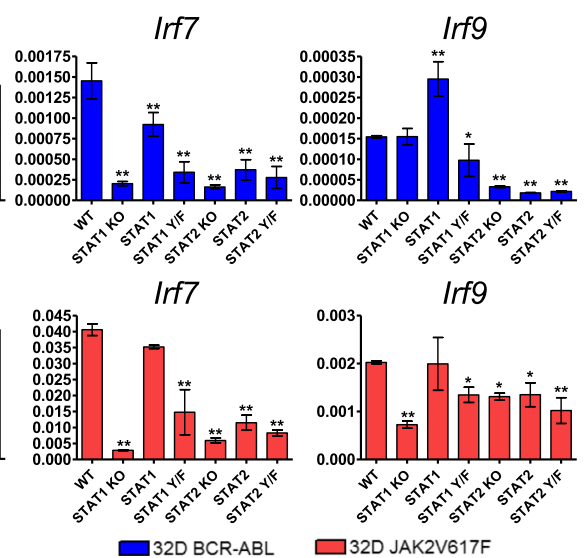

Fig. 4 Reconstitution of 32D-BCR-ABL and 32D-JAK2V617F STAT1ko and STAT2ko cells. 32D-BCR-ABL and 32D-JAK2V617F cells (depicted as $W T$, respectively), which passed through the CRISPR STAT KO process but showed no knockout, were used as control cell lines. 32D-BCR-ABL (a) and 32D-JAK2V617F (b) STAT1 ko or STAT2ko cells reconstituted with wt-STAT1, wt-STAT2, STAT1Y701F (Y/F), or STAT2Y689F (Y/F) were applied in a MTT assay and treated with the indicated concentrations of IFNa $\left(0-10^{4} \mathrm{U} / \mathrm{ml}\right)$ for $72 \mathrm{~h}$. MTT assays have been performed four times in independent experiments, and untreated controls were analyzed with one-way ANOVA and Dunn's multiple comparison test. Further statistical analysis can be found in Additional file 9: Figure S7A, B. c Western blot analysis of the 32D cell lines depicted in a and b treated for $4 \mathrm{~h}$ with $100 \mathrm{U} / \mathrm{ml}$ IFNa or left untreated. Phosphorylation of STAT1, STAT2, and STAT3 was analyzed. GAPDH served as the loading control. $\mathbf{d}$ mRNA expression of interferon-stimulated genes in the indicated cell lines. Stat2 qPCR primer detected the ectopically expressed Stat2 mRNA, explaining the strong upregulation, and endogenous Stat2 can thus not be evaluated in the reconstituted experiments. Gene expression was calculated as a percentage of Gapdh, and the mean values \pm SD are depicted. ${ }^{*} p<0.05,{ }^{* *} p<0.01$, ${ }^{* * *} p<0.001$. The respective $32 \mathrm{D}$ cells were grown in IL3-source-free medium $24 \mathrm{~h}$ before application into the experiment

BCR-ABL- and JAK2V617F-positive cells (Additional file 8: Figure S9A, B).

STAT2 reconstitution (and to a lesser extent, STAT2Y/F) was able to rescue ISG transcription (Stat1 and Irf7, while Irf9 was not decreased in the absence of STAT2) in JAK2V617F- but not BCRABL-positive cells (Fig. 4d). In all of these cells, reconstitution of STAT2 rescued IFNa-stimulated ISG expression at least partially (Additional file 8: Figure S9a, b).
Together, our results support the hypothesis that, in BCR-ABL-expressing cells, STAT2 is a survival factor but not an IFNa-sensitizing factor. In contrast, in JAK2V617F-positive cells, STAT2 is a survival as well as an IFNa-sensitizing factor.

Histone modifications at TFBS in 32D-BCR-ABL and 32DJAK2V617F cells correlate with gene expression Screening for differences in the acetylation profile in 32D-EV 32D-BCR-ABL, or 32D-JAK2V617F was done 
by ChIP-seq experiments to correlate these findings with the expression array data. ChIP-seq analysis revealed specific active regulatory regions (Additional file 11: Figure S10) and a significant enrichment of STAT1, IRF1, and IRF8 binding sites in the acetylated regions in 32D-JAK2V617F compared to 32D-BCR-ABL cells (Fig. 5a; Additional file 1: Table S9).

Based on these findings, validation of H3K9 acetylation (H3K9ac) by ChIP-PCR was performed at the promoter of ISGs at ISRE sites after TKI and/or IFNa treatment. Stat1, Stat2, Irf1, and Irf9 showed significantly higher H3K9ac in 32D-JAK2V617F cells compared to 32D-BCR-ABL cells (Fig. 5b). Interestingly, IFNa reduced the acetylation at the promoter of Stat1, Stat2, Irf1, and Irf9 in 32D-BCR-ABL cells, but increased it in all four promoter regions in 32D-JAK2V617F cells (Fig. 5b). No changes were observed when 32D-BCR-ABL cells were treated with imatinib alone or in combination with IFNa, except for induction of Stat 2 promoter acetylation with the combination treatment. Ruxolitinib treatment inhibited IFNa-induced H3K9ac of all four gene promoter regions in 32D-JAK2V617F cells (Fig. 5b).

However, since IFNa induces high mRNA expression (Fig. 2a) despite lower H3K9ac levels (Fig. 5b) in 32D-BCR-ABL cells, further mechanisms of gene regulation were likely to play a role. Thus, further histone marks were analyzed and compared to ISG mRNA expression patterns (Fig. 5c). The results confirmed close correlation of high mRNA expression with a low abundance of the repressive H3K27me3 chromatin mark but high abundance of active H3K9ac, H3K27ac, and H3K4me3 marks (Fig. 5c).

\section{Discussion}

Since its discovery almost 60 years ago, IFNa and its pegylated form, PegIFNa, have been successfully used therapeutically in various malignancies, including MPN [33]. Nevertheless, the IFNa response is dependent on the MPN subtype, with IFNa monotherapy inducing complete hematologic remission (CHR) rates of $76 \%$ and complete molecular remission rates of $18 \%$ in $\mathrm{Ph}$ neg MPNs such as PV or ET [38], but no more than 55\% CHR rates in CML $[39,40]$ and much lower rates in overt PMF $[41,42]$.

In this study, we compared the IFNa response of BCR-ABL- vs. JAK2V617F-positive cells and their dependence on the transcription factors STAT1 and STAT2. BCR-ABL cells were resistant to growth inhibition and apoptosis by IFNa single treatment, while 32D-JAK2V617F cells were highly sensitive (Fig. 1a). It has been described that BCR-ABL suppresses IFN-inducible gene expression and IFNa responsiveness in Ba/F3-BCR-ABL cells [20]. We confirmed these results and significantly extended the analysis to human cell lines, PBMCs, and CD34+ cells from
BCR-ABL-positive vs. BCR-ABL-negative MPN patients (Figs. 1 and 2). Although CML PBMCs show clear reduction of the analyzed ISGs, supporting our cell line data, the regulation in PV-derived PBMC samples was heterogeneous. This might be explained by the differing allelic burden in PBMC in comparison to $100 \%$ positivity in cell lines. ISG suppression by BCR-ABL may be partially explained by lysosomal degradation of IFNAR1 in BCR-ABL-expressing cells, resulting in reduced IFNa sensitivity [26] and the presence of pY-STAT2, reported to be involved in ISG repression [43].

Using our CRISPR/Cas9n-mediated STAT1 and STAT2 knockout cell lines, we were able to demonstrate that, in BCR-ABL-positive cells, STAT2 is essential for IFNa-induced STAT1 phosphorylation (Fig. 3a), supported by the observation that pY-STAT2 recruits STAT1 to the activated IFNa receptor [44]. Importantly, we demonstrate that, in JAK2V617F-expressing cells, STAT1 phosphorylation by IFNa stimulation is independent from STAT2 (Fig. 3b), suggesting that receptor recruitment is different in JAK2V617F-positive cells. Furthermore, STAT2ko is less effective in repression of ISG expression (Fig. 3f and Additional file 10: Figure S8A, B) in comparison to JAK2V617F-STAT1ko cells, presumably due to the retained pY-STAT1 after IFNa stimulation (Fig. 3b).

In the following, we demonstrate that STAT2Y689F induces STAT1 expression in JAK2V617F-positive cells but not in BCR-ABL-expressing cells (Fig. 4c and Fig. 6). We found higher levels of the negatively regulating histone mark H3K27me3 [45] in BCR-ABL-expressing cells, probably explaining the incapacity to upregulate STAT1 expression. Furthermore, we demonstrated a re-induction of ISGs in BCR-ABL-positive cells after imatinib treatment and a further increase when cells were treated with a combination of TKI and IFNa (Fig. 2), most likely due to positive feedback of re-induced STAT1, loss of negatively regulating histone marks, and rescue of IFNAR1 surface expression after pretreatment of CML cell lines with TKI [26].

Previous reports assessing the mechanism of IFNa resistance in CML patients found both STAT1 deficiency [17] and SOCS3 overexpression [18] to play an important role. However, SOCS3 overexpression was only detected at the blast crisis but not chronic phase stage of CML [18]. Furthermore, while STAT1 expression was well correlated with the in vivo IFNa response in one study [17], BCR-ABL was only found to inhibit STAT1 phosphorylation but not STAT1 protein expression in another study [20]. Indeed, overexpression of STAT1 in our 32D-BCR-ABL cells was only marginally capable to induce IFNa efficacy, both in the presence or absence of endogenous STAT1 (Fig. 4 and Additional file 7: Figure S6). However, STAT2 reconstitution in STAT2-deficient 

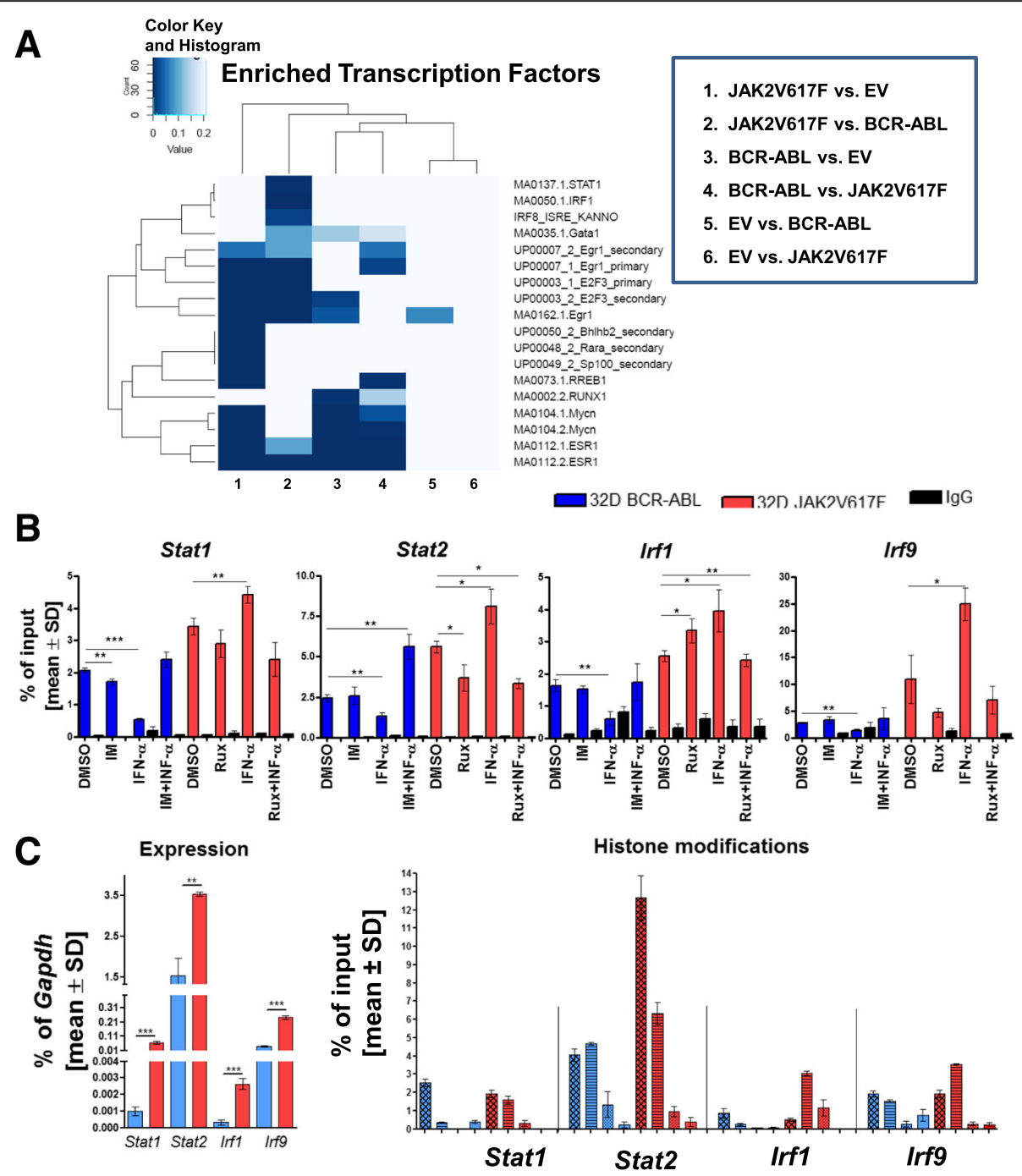

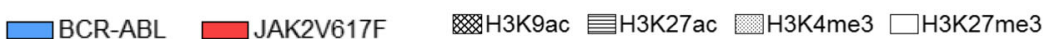

Fig. 5 Histone modifications in the promoter region of interferon target genes differ between 32D-BCR-ABL- and 32D-JAK2V617F-positive cells. a Enrichment analysis of transcription factor binding sites (TFBS) in acetylation (H3K9ac) peaks based on ChIP-seq data. Differentially regulated genes were analyzed for the presence of TFBS in the acetylation peaks, and it was tested if the number of TFBS is significantly different. b 32D-BCR-ABL-transduced (blue) and 32D-JAK2V617F-transduced (red) cells were analyzed for changes of H3K9 acetylation in the promoter region of Stat1, Stat2, Irf1, and Irf9 after $4 \mathrm{~h}$ of $1 \mu \mathrm{M} \mathrm{TKI}$ and/or $100 \mathrm{U} / \mathrm{ml}$ IFNa treatment by ChIP-PCR and were tested for differences in comparison to the corresponding DMSO-treated cell line. ${ }^{*} p<0.05,{ }^{* *} p<0.01,{ }^{* * *} p<0.001$. c Further histone marks such as H3K27 acetylation (active mark), H3K4 tri-methylation (active mark), or H3K27 trimethylation (repressive mark) were measured by ChIP-PCR (right side) and correlated with Stat1, Stat2, Irf1, and Irf9 mRNA expression (left side) in these 32D-BCR-ABL (blue) and 32D-JAK2V617F (red) cells. Binding was calculated as the percentage of input and shown as mean value \pm SD. ChIPs have been performed twice and expression analysis three times in triplicate in independent experiments. Oncogene-expressing 32D cell lines were growing without WEHI for the experimental procedure

BCR-ABL- and JAK2V617F-positive cells increased basal cell viability but did not change upon IFNa treatment (Fig. 4a). It needs to be mentioned that ectopic expression of STAT2 did not necessarily rescue the observed results in STAT2 +/+ cells. Ectopic expression of STAT2 is probably too high, which would suggest high cellular sensitivity to changes in STAT2 expression.

These results suggest that the ratio of different STAT proteins is crucial for cell proliferation, survival, and
IFNa responsiveness in CML. In addition to differential STAT1/STAT2 signaling in the clonal cells themselves, cell-extrinsic effects such as IFNa-mediated activation of the immune system is certainly important [46]. However, in the present study, we specifically focused on the cell-autonomous effects of IFNa.

Contribution of STAT proteins in IFN signaling is very complex as most STATs can form homo- as well as hetero-dimers in phosphorylated and unphosphorylated 


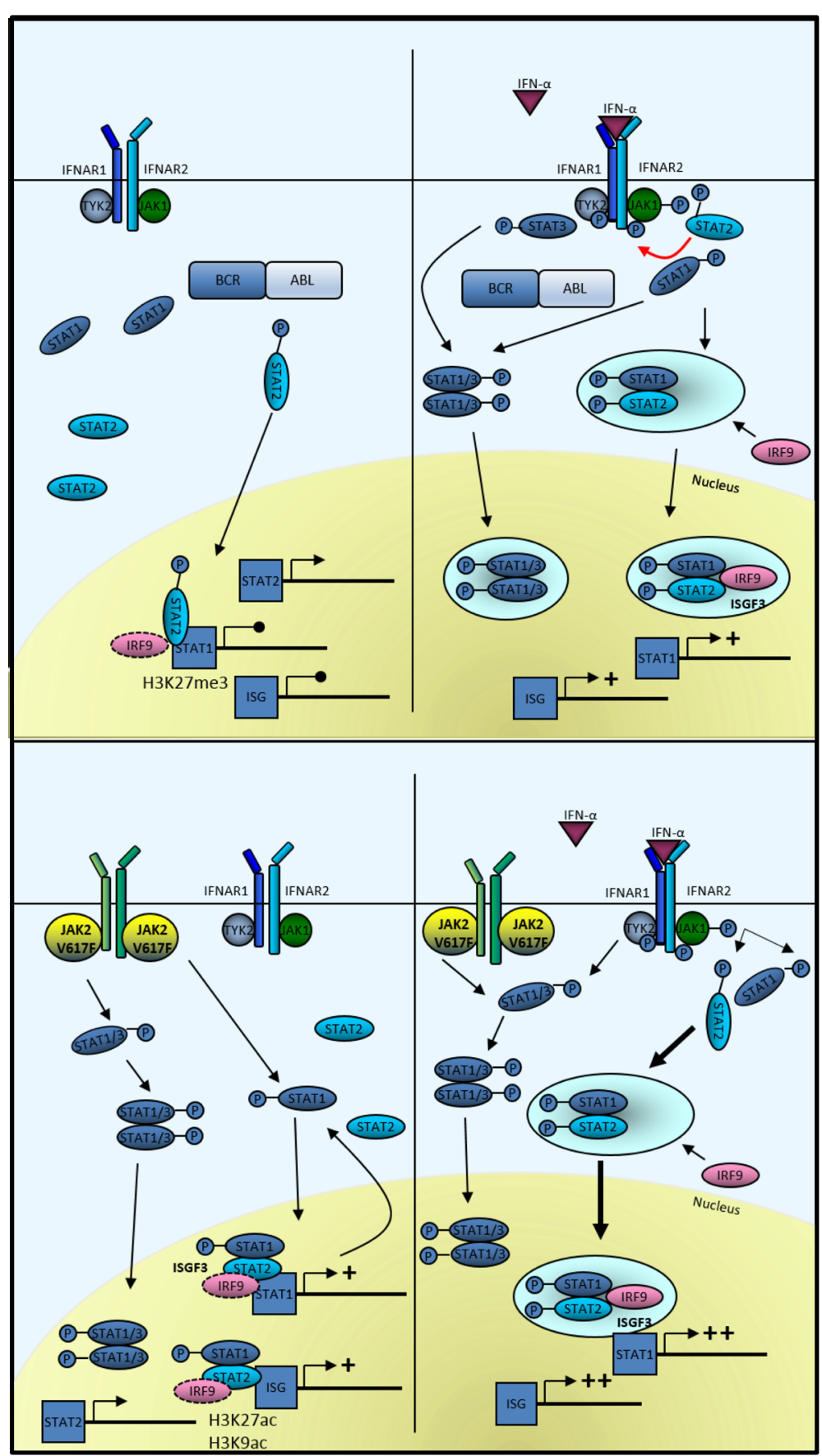

Fig. 6 Simplified overview of ISG regulation by STAT1 and STAT2 in BCR-ABL- and JAK2V617F-positive cells. In BCR-ABL-expressing cells, STAT2 is partially phosphorylated leading to ISG repression [43] and is not capable to induce STAT1 expression. In addition, chromatin marks negatively regulating gene expression are present (i.e., H3K27me3). STAT2, although not phosphorylated in JAK2V617F-positive cells, can induce STAT1 expression in the presence of histone marks representing active promoters (i.e., H3K9ac and H3K27ac). Upon stimulation with IFNa, STAT2 is essential for STAT1 phosphorylation at its tyrosine residue Y701 in BCR-ABL-positive cells (indicated by red arrow). The ISGF3 complex is formed and ISG expression is induced. IFNa stimulation of JAK2V617F-positive cells leads to ISGF3 complex formation and ISG as well as STAT1 promoter access. The equilibrium of STAT1/STAT1 and STAT1/STAT3 dimers shifts in dependence of the amount of active STAT2. STAT1/3 illustrates different dimer options: STAT1/STAT1, STAT1/STAT3, and STAT3/STAT3. In BCR-ABL-expressing cells, STAT3 is phosphorylated after IFNa binding to its receptor. P, tyrosine phosphorylation 
state, thereby activating distinct subsets of genes [47, 48]. The expression level of STAT3 and its phosphorylation is crucial for the STAT1 transcriptional profile, as higher (phospho)-STAT3 levels reduce the amount of STAT1 homodimers and at the same time support the ISGF3dependent gene expression [49], thereby influencing IFNa responsiveness. STAT3 was only phosphorylated in 32D-JAK2V617F cells but not in BCR-ABL-expressing cells (Fig. 2c), therefore supporting ISGF3 complex formation. Surprisingly, 32D-JAK2V617F-S1ko-STAT1Y/F cells were still responsive to IFNa (Fig. $4 \mathrm{~b}$ and Additional file 10: Figure S8), suggesting the ISGF3 complex formation even without phosphorylation of STAT1 at its regulatory tyrosine 701.

Re-expression of STAT2 and STAT2YF led to increased viability of BCR-ABL- and JAK2V617F-positive S2ko cells. Nevertheless, only JAK2V617F-S2ko-STAT2(Y/F) cells were sensitized to low doses of IFNa, presumably due to upregulation of STAT1 protein expression (Fig. 4a, b). At the same time, while targeted disruption of STAT2 reduced basal viability of JAK2V617F cells, IFNa treatment increased cell viability, possibly through hyper-activation of STAT3 and reduced negative regulation (Figs. 3d and 4c). Intriguingly, ectopic expression of STAT2(Y/F) did not decrease PY-STAT3 to normal levels in 32D-JAK2V617F-S2ko cells (Fig. 4c), which was in line with only partial reconstitution of ISG induction (Additional file 8: Figure S9a and b) [50] and suggested that JAK2V617F but not BCR-ABL alters the requirement for STAT2 in the response to IFNa. These results argue for a differential and significant role of STAT2 but also STAT3 in BCR-ABL- vs. JAK2V617F-expressing cells (Fig. 6). In addition, our data also indicate a leading role for PY-STAT1 and STAT2, as a regulator of STAT1 transcription, in JAK2V617F-expressing cells for induction of apoptosis.

STAT binding at the promoter of ISGs is strongly influenced by histone modifications altering promoter accessibility. In former studies, it was demonstrated that histone acetylation is important for hematopoietic differentiation, which can be counteracted by overexpression of HDAC1 [51]. Our H3K9ac ChIP-seq data revealed an increase of acetylation at the binding sites of interferonregulated transcription factors in line with an increase of IFNa target genes in JAK2V617F-positive cells, providing a link between acetylation and active gene transcription. In addition, STAT1 and STAT2 have been associated with histone acetyltransferases, resulting in localized acetylation of histones [52, 53]. Our data lead to the conclusion that acetylation at the promoter of ISGs in 32D-JAK2V617F but not 32D-BCR-ABL cells can be correlated with gene expression, with or without a response to TKI and IFNa treatment. HDAC inhibitors showed selective inhibition of ISGs that are activated by STAT1 and STAT2 [54]. In addition, IRF9 was shown to be important for the binding of RNA polymerase II to the promoter of these target genes to modulate the
HDAC function and gene expression. Analysis of additional histone modifications confirmed a complex time and cell type-specific mechanism of gene regulation, as the presence of different modifications correlated with mRNA levels in the analyzed cells (Fig. 5c).

\section{Conclusions}

Taken together, in BCR-ABL-positive cells, STAT2 was found to be a survival factor but not an IFNa-sensitizing factor, indicating an important role of STAT2 protein in the prediction of responsiveness of CML patients to IFNa therapy. In contrast, in JAK2V617F-positive cells, STAT2 is a survival and an IFNa-sensitizing factor, regulating STAT1 expression even in its unphosphorylated state (Fig. 6). Therefore, the complex network of STAT1, STAT2, and STAT3 in their unphosphorylated and phosphorylated states could probably help to predict the response to IFNa. Moreover, our data suggest that the analysis of the composition of histone modifications may be useful for the prediction of gene expression and response to therapy.

\section{Additional files}

Additional file 1: Supplementary Material \& Methods. Table S1. Primer for cloning STAT1 and STAT2 with included restriction sites. Table S2. STAT1 and STAT2 primers for mutagenesis. Table S3. RT-qPCR primer for gene expression analysis. Table S4. Western blot antibodies. Table S5. gRNA Oligonucleotides. Table S6. ChIP antibodies. Table S7. ChIP-PCR Primer. Table S8. GSEA of a set of 45 interferon-stimulated genes (ISG). Table S9. Statistics for the enrichment analysis of transcription factor binding sites (TFBS) in acetylation (H3K9ac) peaks. (DOCX 44 kb)

Additional file 2: Figure S1. Knockout of STAT1 or STAT2 with CRISPR/ Cas9n technology. Four guide RNAs have been generated for STAT1 or STAT2 knockout in 32D-BCR-ABL and 32D-JAK2V617F cells, respectively. Excised exons are given. (PDF $19 \mathrm{~kb}$ )

Additional file 3: Figure S2. MTT assay of 32D-BCR-ABL and 32D-JAK2V617F cells treated with IFNa. 32D-BCR-ABL-(blue) and 32D-JAK2V617F-(red) positive cells were treated with IFNa $\left(0-10^{4} \mathrm{U} / \mathrm{ml}\right)$ alone (continuous lines) or in combination with $0.1 \mu \mathrm{M}$ imatinib (IM) or ruxolitinib (Rux) (dotted lines) for $72 \mathrm{~h}$ and the viability was measured by MTT. Viability was normalized to the untreated control and mean values \pm SD are depicted. The respective 32D cells were WEHI starved for $24 \mathrm{~h}$ before starting the experiments. Experiments were performed in triplicate and conducted three times. (PDF $27 \mathrm{~kb}$ )

Additional file 4: Figure S3. BCR-ABL reduces ISG expression in 32D cells. Gene expression microarray analysis of 32D-EV, 32D-BCR-ABL, or 32D-JAK2V617F cells. Fold change of gene expression is shown, depicting downregulation of the analyzed gene in blue and upregulation in red. (PDF $134 \mathrm{~kb}$ )

Additional file 5: Figure S4. Effect of extrinsic soluble factors on gene expression in 32D-EV- or 32D-JAK2V617F-positive cells. Supernatant of WEHI-starved 32D-EV- or 32D-JAK2V617F-positive cells was generated overnight, and after removal of the cells, fresh EV (green) or JAK2V617F(red) positive cells were incubated with the supernatant for $2 \mathrm{~h}$ prior to RNA extraction to analyze the expression of IFN target genes. Mean \pm SD values are shown as \% of Gapdh. Independent experiments were performed three times and in triplicate, respectively. (PDF $25 \mathrm{~kb}$ )

Additional file 6: Figure S5. Correlation of ISG expression and JAK2V617F allelic burden and Western blot of 32D EV, BCR-ABL, or JAK2V617F cells. A, ISG expression (\% of GAPDH) and JAK2V617F allelic 
burden (in \%) were plotted against each other and a significant correlation was only found for IRF9 $(p=0.0492)$. Samples with additional mutations (TET2, ASXL1, or EZH2) were highlighted with a red circle. B, Western blot analysis of pY-STAT1 and overall STAT1 protein after TKI and/or IFNa treatment (4 h) of 32D EV (only -/+ IFNa), BCR-ABL, or JAK2V617F cells. All cell lines were starved of WEHI (source of IL-3) for $24 \mathrm{~h}$ before treatment. GAPDH served as the loading control. The same Western blot is shown in Fig. 2c lacking 32D EV cells. (PDF 74 kb)

Additional file 7: Figure S6. Confirmation of successful STAT1 or STAT2 knockout. Western blotting of several 32D-BCR-ABL or 32D-JAK2V617F STAT1 or STAT2 knockout clones. STAT2 antibody was used to confirm the knockout, and GAPDH served as the loading control. 32D cells were WEHI starved for $24 \mathrm{~h}$ before starting the experiment. wt - wild-type clones, ko - knockout clones, het - presumed heterozygous clones (PDF $134 \mathrm{~kb}$ )

Additional file 8: Figure S9. Full RT-qPCR panels of tested ISGs. Illustration of the RT-qPCR results of 32D-BCR-ABL- and 32D-JAK2V617F-WT or -STATko or -STAT1(Y/F) and STAT2(Y/F) reconstituted cell clones treated with IFNa $(100 \mathrm{U} / \mathrm{ml})$ or left untreated (triplicate), corresponding to the data given in Figs. $3 f$ and $4 d$. (a) Stat 1 and Stat2, (b) Irf7 and Irf9. Stat2 QPCR primer detected the ectopically expressed Stat2 mRNA, explaining the strong upregulation, and endogenous Stat2 can thus not be evaluated in the reconstituted experiments (gray bars). Independent experiments were performed three times. (PDF $56 \mathrm{~kb}$ )

Additional file 9: Figure S7. Comparison of CRISPR/Cas9 manipulated $32 \mathrm{D}$ cell lines treated with $100 \mathrm{U}$ IFNa in survival and titration of lower IFNa dosages. Indicated (A) 32D-BCR-ABL and (B) 32D-JAK2V617F cell lines were analyzed in an MTT assay and treated with $100 \mathrm{U}$ IFNa for $72 \mathrm{~h}$ (abstracted from Fig. 4a, b). Absorption was normalized to untreated control cells and statistically analyzed using a $t$ test. Mean values \pm SD are indicated. ${ }^{*} p<0.05,{ }^{* *} p<0.01,{ }^{* * *} p<0.001$. C, 32D-JAK2V617F cells, -S1 ko and S2ko cells, reconstituted with STAT1 (S1RE), STAT1Y701F (S1YF), STAT2 (S2RE), or STAT2Y689F were treated with IFNa $(0,1,5,10$ and 100 $\mathrm{U} / \mathrm{ml}$ ) for $72 \mathrm{~h}$, and the viability was measured by MTT. Mean values \pm SD are depicted. Independent experiments were performed three times and in triplicate, respectively. The respective 32D cells were WEHI starved for $24 \mathrm{~h}$ before starting the experiments. (PDF $26 \mathrm{~kb}$ )

Additional file 10: Figure S8. Illustration of PY-STAT1 in 32DJAK2V617F cells in the absence of IFNa. STAT1 and pY-STAT1 stainings of Fig. 4C, shown with increased contrast. The arrows are indicating the pY-STAT1 bands in 32D-BCR-ABL- and 32D-JAK2V617F-STATko cells. (PDF $203 \mathrm{~kb})$

Additional file 11: Figure S10. H3K9 acetylation profile at ISGs. The ChIP-seq acetylation was visualized with the Integrative Genomics Viewer (IGV). Shown are the H3K9 acetylation peaks at the genomic regions of Irf1, Irf17, Irf9, and Stat1 in 32D-JAK2V7F (JAK2V617F) (red), 32D-BCR-ABL (blue), and 32D-EV (green). (PDF $108 \mathrm{~kb}$ )

\section{Abbreviations}

ChIP: Chromatin immunoprecipitation; CHR: Complete hematologic remission; CML: Chronic myeloid leukemia; ET: Essential thrombocythemia; EV: Empty vector; GEP: Gene expression profiling; GSEA: Gene enrichment analysis; IFNa: Interferon alpha; IFNAR: Interferon alpha receptor; IM: Imatinib; IRF: Interferon responsive factor; ISG: IFN-stimulated genes; ISGF3: IFN-stimulated gene factor 3; ISRE: IFN-stimulated response element; JAK: Janus kinase; KO: Knockout; MPN: Myeloproliferative neoplasms; PBMC: Peripheral blood mononuclear cells; pegIFNa: Pegylated IFNa; PMF: Primary myelofibrosis; PV: Polycythemia vera; Rux: Ruxolitinib; SD: Standard deviation; SOCS: Suppressor of cytokine signaling; STAT: Signal transducer and activator of transcription; TKI: Tyrosine kinase inhibitor

\section{Acknowledgements}

Biomaterial samples were provided by the RWTH centralized Biomaterial Bank Aachen (RWTH CBMB, Aachen, Germany) in accordance with the regulations of the biomaterial bank and the approval of the ethics committee of the medical faculty, RWTH Aachen. We thank Stefan Frank and Boris Greber for vectors. This work was part of the PhD thesis of C.S.

\section{Funding}

This work was in part funded by research grants from the DFG KO2155/6-1 (S.K.), START grant by the Faculty of Medicine in Aachen (N.C.), the Interdisciplinary Center for Clinical Research (IZKF) Aachen (S.K.), Deutsche José Carreras Leukämie-Stiftung (DJCLS) R10/23 (S.K.), Interdisciplinary Center for Clinical Research (IZKF) Aachen Research Group (I.C.), and the Excellence Initiative of the German Federal and State Governments and the German Research Foundation (grant GSC 111) (I.C.).

\section{Availability of data and materials}

All data generated or analyzed during this study are included in this published article [and its supplementary information files]. Datasets analysed during the current study are available at NCBI, GEO DataSets (Accession: GSE5550; GSE120362).

\section{Authors' contributions}

CS designed the research, performed the experiments, analyzed the data, and wrote the paper. MA, TM, and IGC analyzed the data sets and generated the figures. ST, KF, and JB performed the experiments and analyzed the data. DBL performed the ChIP-seq and analyzed the data. MS and THB analyzed the data and contributed in writing the manuscript. NC and SK designed the research, analyzed the data, and corrected the manuscript. All authors approved the final version of the manuscript.

\section{Ethics approval and consent to participate}

Primary patient samples were obtained from patients of the Department of Hematology, Oncology, Hemostaseology, and Stem Cell Transplantation at RWTH Aachen University after written informed consent, as approved by the local ethics committee (EK 127/12 and EK 206/09).

\section{Consent for publication}

Not applicable.

\section{Competing interests}

SK has been a member of Advisory Boards for Pfizer, Incyte/Ariad, Novartis, AOP, BMS, and CTI; receives honoraria and travel funding from Novartis, BMS, Pfizer, Incyte/Ariad, and Shire; has received scientific research support from Novartis Foundation, BMS, and Novartis; and has received travel funding by Alexion. THB reports grants from Pfizer and Novartis and served as an advisor for Novartis, Pfizer, and ARIAD during the conduct of the study. The remaining authors declare that they have no competing interests.

\section{Publisher's Note}

Springer Nature remains neutral with regard to jurisdictional claims in published maps and institutional affiliations.

\section{Author details}

${ }^{1}$ Department of Hematology, Oncology, Hemostaseology, and Stem Cell Transplantation, Faculty of Medicine, RWTH Aachen University, Pauwelsstr 30, 52074 Aachen, Germany. ${ }^{2}$ Institute for Computational Genomics, Faculty of Medicine, RWTH Aachen University, Aachen, Germany. ${ }^{3}$ Regulation of Cellular Differentiation Group, Division of Epigenomics and Cancer Risk Factors, German Cancer Research Center (DKFZ), Heidelberg, Germany.

Received: 1 December 2018 Accepted: 13 March 2019

Published online: 02 April 2019

\section{References}

1. Sawyers CL. Chronic myeloid leukemia. N Engl J Med. 1999;340(17):1330-40.

2. James C, Ugo V, Le Couedic JP, Staerk J, Delhommeau F, Lacout C, et al. A unique clonal JAK2 mutation leading to constitutive signalling causes polycythaemia vera. Nature. 2005;434(7037):1144-8.

3. Levine RL, Wadleigh M, Cools J, Ebert BL, Wernig G, Huntly BJ, et al. Activating mutation in the tyrosine kinase JAK2 in polycythemia vera, essential thrombocythemia, and myeloid metaplasia with myelofibrosis. Cancer Cell. 2005;7(4):387-97.

4. Barnes DJ, Melo JV. Primitive, quiescent and difficult to kill: the role of nonproliferating stem cells in chronic myeloid leukemia. Cell Cycle. 2006;5(24):2862-6. 
5. Jiang X, Zhao Y, Smith C, Gasparetto M, Turhan A, Eaves A, et al. Chronic myeloid leukemia stem cells possess multiple unique features of resistance to BCR-ABL targeted therapies. Leukemia. 2007;21(5):926-35.

6. Hochhaus A, O'Brien SG, Guilhot F, Druker BJ, Branford S, Foroni L, et al. Six-year follow-up of patients receiving imatinib for the first-line treatment of chronic myeloid leukemia. Leukemia. 2009;23(6):1054-61.

7. Harrison C, Kiladjian JJ, Al-Ali HK, Gisslinger H, Waltzman R, Stalbovskaya V, et al. JAK inhibition with ruxolitinib versus best available therapy for myelofibrosis. N Engl J Med. 2012;366(9):787-98.

8. Verstovsek S, Mesa RA, Gotlib J, Levy RS, Gupta V, DiPersio JF, et al. A double-blind, placebo-controlled trial of ruxolitinib for myelofibrosis. N Engl J Med. 2012;366(9):799-807.

9. Vannucchi AM. Ruxolitinib versus standard therapy for the treatment of polycythemia vera. N Engl J Med. 2015;372(17):1670-1.

10. Corbin AS, Agarwal A, Loriaux M, Cortes J, Deininger MW, Druker BJ. Human chronic myeloid leukemia stem cells are insensitive to imatinib despite inhibition of BCR-ABL activity. J Clin Invest. 2011;121(1):396-409.

11. Hamilton A, Helgason GV, Schemionek M, Zhang B, Myssina S, Allan EK, et al. Chronic myeloid leukemia stem cells are not dependent on Bcr-Abl kinase activity for their survival. Blood. 2012;119(6):1501-10.

12. El Eit RM, Iskandarani AN, Saliba JL, Jabbour MN, Mahfouz RA, Bitar NM, et al. Effective targeting of chronic myeloid leukemia initiating activity with the combination of arsenic trioxide and interferon alpha. Int J Cancer. 2014;134(4):988-96.

13. Hasan S, Lacout C, Marty C, Cuingnet M, Solary E, Vainchenker W, et al. JAK2V617F expression in mice amplifies early hematopoietic cells and gives them a competitive advantage that is hampered by IFNalpha. Blood. 2013;122(8):1464-77.

14. Mullally A, Bruedigam C, Poveromo L, Heidel FH, Purdon A, Vu T, et al. Depletion of Jak2V617F myeloproliferative neoplasm-propagating stem cells by interferon-alpha in a murine model of polycythemia vera. Blood. 2013;121(18):3692-702.

15. Ivashkiv LB, Donlin LT. Regulation of type I interferon responses. Nat Rev Immunol. 2014;14(1):36-49.

16. Tanabe Y, Nishibori T, Su L, Arduini RM, Baker DP, David M. Cutting edge: role of STAT1, STAT3, and STAT5 in IFN-alpha beta responses in T Iymphocytes. J Immunol. 2005;174(2):609-13.

17. Landolfo S, Guarini A, Riera L, Gariglio M, Gribaudo G, Cignetti A, et al. Chronic myeloid leukemia cells resistant to interferon-alpha lack STAT1 expression. Hematol J. 2000;1(1):7-14

18. Sakai I, Takeuchi K, Yamauchi H, Narumi H, Fujita S. Constitutive expression of SOCS3 confers resistance to IFN-alpha in chronic myelogenous leukemia cells. Blood. 2002;100(8):2926-31.

19. Grumbach IM, Mayer IA, Uddin S, Lekmine F, Majchrzak B, Yamauchi $H$, et al. Engagement of the CrkL adaptor in interferon alpha signalling in BCR-ABL-expressing cells. Br J Haematol. 2001;112(2):327-36.

20. Katsoulidis E, Sassano A, Majchrzak-Kita B, Carayol N, Yoon P, Jordan A, et al. Suppression of interferon (IFN)-inducible genes and IFN-mediated functional responses in BCR-ABL-expressing cells. J Biol Chem. 2008;283(16):10793-803.

21. Platanias LC. Mechanisms of type-I- and type-II-interferon-mediated signalling. Nat Rev Immunol. 2005;5(5):375-86.

22. Mayer IA, Verma A, Grumbach IM, Uddin S, Lekmine F, Ravandi F, et al. The p38 MAPK pathway mediates the growth inhibitory effects of interferon-alpha in BCR-ABL-expressing cells. J Biol Chem. 2001;276(30):28570-7.

23. Lu M, Zhang W, Li Y, Berenzon D, Wang X, Wang J, et al. Interferon-alpha targets JAK2V617F-positive hematopoietic progenitor cells and acts through the p38 MAPK pathway. Exp Hematol. 2010;38(6):472-80.

24. Saleiro D, Mehrotra S, Kroczynska B, Beauchamp EM, Lisowski $P$, Majchrzak-Kita B, et al. Central role of ULK1 in type I interferon signaling. Cell Rep. 2015;11(4):605-17.

25. Mehrotra S, Sharma B, Joshi S, Kroczynska B, Majchrzak B, Stein BL, et al. Essential role for the Mnk pathway in the inhibitory effects of type I interferons on myeloproliferative neoplasm (MPN) precursors. J Biol Chem. 2013;288(33):23814-22.

26. Bhattacharya S, Zheng H, Tzimas C, Carroll M, Baker DP, Fuchs SY. Bcr-abl signals to desensitize chronic myeloid leukemia cells to IFNalpha via accelerating the degradation of its receptor. Blood. 2011;118(15):4179-87.

27. Maxwell BL, Talpaz M, Gutterman JU. Down-regulation of peripheral blood cell interferon receptors in chronic myelogenous leukemia patients undergoing human interferon (HulFN alpha) therapy. Int J Cancer. 1985;36(1):23-8.
28. Han L, Schubert C, Kohler J, Schemionek M, Isfort S, Brummendorf TH, et al Calreticulin-mutant proteins induce megakaryocytic signaling to transform hematopoietic cells and undergo accelerated degradation and Golgi-mediated secretion. J Hematol Oncol. 2016;9(1):45.

29. Elling C, Erben P, Walz C, Frickenhaus M, Schemionek M, Stehling M, et al. Novel imatinib-sensitive PDGFRA-activating point mutations in hypereosinophilic syndrome induce growth factor independence and leukemia-like disease. Blood. 2011;117(10):2935-43.

30. Ran FA, Hsu PD, Lin CY, Gootenberg JS, Konermann S, Trevino AE, et al Double nicking by RNA-guided CRISPR Cas9 for enhanced genome editing specificity. Cell. 2013;154(6):1380-9.

31. Schemionek M, Kharabi Masouleh B, Klaile Y, Krug U, Hebestreit K, Schubert $C$, et al. Identification of the adapter molecule MTSS1 as a potential oncogene-specific tumor suppressor in acute myeloid leukemia. PLoS One. 2015;10(5):e0125783.

32. Schafer M, Lkhagvasuren O, Klein HU, Elling C, Wustefeld T, Muller-Tidow C, et al. Integrative analyses for omics data: a Bayesian mixture model to assess the concordance of ChIP-chip and ChIP-seq measurements. J Toxicol Environ Health A. 2012;75(8-10):461-70.

33. Bekisz J, Baron S, Balinsky C, Morrow A, Zoon KC. Antiproliferative properties of type I and type I| interferon. Pharmaceuticals. 2010;3(4):994-1015.

34. Diaz-Blanco E, Bruns I, Neumann F, Fischer JC, Graef T, Rosskopf M, et al. Molecular signature of CD34(+) hematopoietic stem and progenitor cells of patients with CML in chronic phase. Leukemia. 2007;21(3):494-504.

35. Varemo L, Nielsen J, Nookaew I. Enriching the gene set analysis of genome-wide data by incorporating directionality of gene expression and combining statistical hypotheses and methods. Nucleic Acids Res. 2013;41(8):4378-91.

36. Wright HL, Thomas HB, Moots RJ, Edwards SW. Interferon gene expression signature in rheumatoid arthritis neutrophils correlates with a good response to TNFi therapy. Rheumatology. 2015;54(1):188-93.

37. Czech J, Cordua S, Weinbergerova B, Baumeister J, Crepcia A, Han L, Maié T, Costa IG, Denecke B, Maurer A, Schubert C, Feldberg K, Gezer D, Brümmendorf TH, Müller-Newen G, Mayer J, Racil Z, Kubesova B, Knudsen T, Sørensen AL, Holmström M, Kjær L, Skov V, Larsen TS, Hasselbalch HC, Chatain N, Koschmieder S. JAK2V617F but not CALR mutations confer increased molecular responses to interferon-a via JAK1/STAT1 activation. Leukemia. 2018. https://doi.org/10.1038/s41375-018-0295-6. Epub ahead of print.

38. Quintas-Cardama A, Abdel-Wahab O, Manshouri T, Kilpivaara O, Cortes J, Roupie $\mathrm{AL}$, et al. Molecular analysis of patients with polycythemia vera or essential thrombocythemia receiving pegylated interferon alpha-2a. Blood. 2013;122(6):893-901.

39. Guilhot F, Chastang C, Michallet M, Guerci A, Harousseau JL, Maloisel F, et al. Interferon alfa-2b combined with cytarabine versus interferon alone in chronic myelogenous leukemia. French Chronic Myeloid Leukemia Study Group. N Engl J Med. 1997;337(4):223-9.

40. O'Brien SG, Guilhot F, Larson RA, Gathmann I, Baccarani M, Cervantes F, et al. Imatinib compared with interferon and low-dose cytarabine for newly diagnosed chronic-phase chronic myeloid leukemia. N Engl J Med. 2003;348(11):994-1004

41. Gowin K, Thapaliya P, Samuelson J, Harrison C, Radia D, Andreasson B, et al. Experience with pegylated interferon alpha-2a in advanced myeloproliferative neoplasms in an international cohort of 118 patients. Haematologica. 2012;97(10):1570-3.

42. lanotto JC, Chauveau A, Boyer-Perrard F, Gyan E, Laribi K, Cony-Makhoul P, et al. Benefits and pitfalls of pegylated interferon-alpha2a therapy in patients with myeloproliferative neoplasm-associated myelofibrosis: a French Intergroup of Myeloproliferative neoplasms (FIM) study. Haematologica. 2018;103(3):438-46.

43. Testoni B, Vollenkle C, Guerrieri F, Gerbal-Chaloin S, Blandino G, Levrero M Chromatin dynamics of gene activation and repression in response to interferon alpha (IFN (alpha)) reveal new roles for phosphorylated and unphosphorylated forms of the transcription factor STAT2. J Biol Chem. 2011;286(23):20217-27

44. Leung S, Qureshi SA, Kerr IM, Darnell JE Jr, Stark GR. Role of STAT2 in the alpha interferon signaling pathway. Mol Cell Biol. 1995;15(3):1312-7.

45. Conway E, Healy E, Bracken AP. PRC2 mediated H3K27 methylations in cellular identity and cancer. Curr Opin Cell Biol. 2015;37:42-8.

46. Burchert A, Wolfl S, Schmidt M, Brendel C, Denecke B, Cai D, et al. Interferon-alpha, but not the ABL-kinase inhibitor imatinib (STI571), induces 
expression of myeloblastin and a specific T-cell response in chronic myeloid leukemia. Blood. 2003;101(1):259-64.

47. Blaszczyk K, Nowicka H, Kostyrko K, Antonczyk A, Wesoly J, Bluyssen HA. The unique role of STAT2 in constitutive and IFN-induced transcription and antiviral responses. Cytokine Growth Factor Rev. 2016;29:71-81.

48. Park HJ, Li J, Hannah R, Biddie S, Leal-Cervantes Al, Kirschner K, et al. Cytokine-induced megakaryocytic differentiation is regulated by genome-wide loss of a uSTAT transcriptional program. EMBO J. 2016:35(6):580-94.

49. Ho HH, Ivashkiv LB. Role of STAT3 in type I interferon responses. Negative regulation of STAT1-dependent inflammatory gene activation J Biol Chem. 2006;281(20):14111-8.

50. Wang WB, Levy DE, Lee CK. STAT3 negatively regulates type I IFN-mediated antiviral response. J Immunol. 2011;187(5):2578-85.

51. Wada T, Kikuchi J, Nishimura N, Shimizu R, Kitamura T, Furukawa Y. Expression levels of histone deacetylases determine the cell fate of hematopoietic progenitors. J Biol Chem. 2009;284(44):30673-83.

52. Zhang JJ, Vinkemeier U, Gu W, Chakravarti D, Horvath CM, Darnell JE Jr. Two contact regions between Stat 1 and CBP/p300 in interferon gamma signaling. Proc Natl Acad Sci U S A. 1996;93(26):15092-6.

53. Paulson M, Press $\mathrm{C}$, Smith E, Tanese N, Levy DE. IFN-stimulated transcription through a TBP-free acetyltransferase complex escapes viral shutoff. Nat Cell Biol. 2002;4(2):140-7.

54. Sakamoto S, Potla R, Larner AC. Histone deacetylase activity is required to recruit RNA polymerase II to the promoters of selected interferon-stimulated early response genes. J Biol Chem. 2004;279(39):40362-7.

Ready to submit your research? Choose BMC and benefit from:

- fast, convenient online submission

- thorough peer review by experienced researchers in your field

- rapid publication on acceptance

- support for research data, including large and complex data types

- gold Open Access which fosters wider collaboration and increased citations

- maximum visibility for your research: over $100 \mathrm{M}$ website views per year

At $\mathrm{BMC}$, research is always in progress.

Learn more biomedcentral.com/submissions 